\title{
PREPARATION AND SOME GALVANOMAGNETICS PROPERTIES OF GdCo MULTILAYER FILMS
}

\author{
A. N. SOROKIN and A. V. SVALOV \\ Institute Physics and Applied Mathematics, Ural State University, Ekaterinburg, Russia
}

\begin{abstract}
The multilayer amorphous GdCo films were prepared by diode and triode r.f. sputtering method. The compositions of the layers were determined by a magnetic compensation temperature $\left(T_{\text {comp }}\right)$ with use of torque method. $T_{\text {camp }}$ was found by the dependence torque moment as a function temperature $L(T)$. The minimums of this function were connected by $T_{\text {comp. }}$. The magnetization reversal processes were studied by use of Hall effect and magnetoresistance (MR) methods. The hysteresis. loops of anomalous Hall effect and magnetoresistance have three satellites loops besides of the band loop. The observed anomalous hysteresis loops of Hall effect and MR are discussed in terms double layers' model with use the H-T diagram of the magnetic state.
\end{abstract}

KEYWORDS: MULTILAYER FILMS, COMPENSATION TEMPERATURE, HYSTERESIS LOOPS, SWITCHING FIELD.

\section{INTRODUCTION}

The attention to the preparation and the investigation of the properties of the exchange coupled multilayer films of rare earth-transition metal (RE-TM) is due to the possibility of their application as a carrier for magneto-optical disks (1). It was found formerly that the amorphous double layer films has. magnetic properties differ from properties of single layer films. The behavior of the magneto-optical effects and galvanomagnetics effects have been explained in terms exchange coupling between of the layers (2). In particular, the additional studies are necessary in order to better interpretation of the reversal magnetization processes in double and multilayer RE-TM films. For the preparation of the multilayer films by r.f. sputtering two different argon pressures (3) and bias voltage sputtering (4) can be use. In this report, some results by the sputtering and the investigation of the properties of multilayer Gd-Co films will be presented. We are applied the specially work out methods of the preparation multilayer films (5) what can be improved also essential uniform of the composition films on the area and the thickness (6). The films prepared by bias voltage sputtering have been used for the confirmation of the different composition in layers of the films, which were produced by the developed new method (5). The compensation temperature $T_{\text {comp }}$ wherein magnetizations of the two sublattices RE and TM are equal and opposite to each other, we try to use as the main parameter for control of the layers composition ( $\mathcal{T}$ ). The data by $T_{\text {compp }}$ correspond to the magnetic active parts of the film composition. In order to obtain the information about the magnetic state of the 'multilayer films the several measurements have been utilized. One method is the measurement of the extraordinary or anomalous Hall effect as a function of applied magnetic field at different temperatures. It is known, that the anomalous Hall effect in amorphous magnetic materials is very large in comparison to the ordinary. The RE and TM sublatice carry in deposit in the measured Hall voltage. The deposit of the $\mathrm{TM}$ sublatice is more than deposit of the RE sublatice (8). H-T diagram of the magnetic state is used for an explanation of discovered anomaly in the hysteresis loops of Hall effect and magnetoresistance.

\section{EXPERIMENTAL}

The Gd-Co multilayer films were deposited onto water glass substrates by diode and triode r.f. $(13,56 \mathrm{MHz})$ sputtering at the argon sputtering pressure $1-2 \cdot 10^{-1}$ Torr. The target was in the form of the $120 \mathrm{~mm}$ diameter cobalt disc partly by uniform distributed $4 \mathrm{~mm}$ diameter gadolinium chips. These chips were pressed in a cobalt plate. The total input power was $1.5 \mathrm{~kW}$. The deposition rate was 10 and $25 \mathrm{~nm} / \mathrm{min}$. The target-substrate distance was $80 \mathrm{~mm}$. A d.c. current of the discharge was 4A for triode r.f. sputtering methods. The temperature measurements of the torque moment (L) were fulfilled by the aid of torque magnetometer. The magnetic field was directed at $45^{\circ}$ to the specimen surface. A standard d.c. technique with a measuring current of $1 \mathrm{~mA}$ was used for Hall voltage and magnitoresistanse measurements. The resulting voltage was plotted simultaneously on X-X recorder as a function of magnetic field at different temperature. The maximum field of $18 \mathrm{kOe}$ was applied perpendicular to the sample. The temperature dependence of the galvanomagnetic effect also was studied. The change of the layers compositions was made by two ways: traditional, i.e.- with the use of the transitory application of the bias voltage to the substrate during sputtering (5-layer film was prepared onto ground of the substrate, $V=0$ ) and special aroused method (5). The composition of the layer in films was defined by the temperature $T_{\text {comp }}$ (7). The compensation temperature in the layers can be determined by minimum torque moment at the dependence $L(T)$. Fig. 1 shows as example of the determination of the films composition with the use of $T_{\text {comp }}$ as a function of the radial position. The curve 1 correspond $T_{\text {comp }}$ of the films produced by the target with gradient of the gadolinium gradient along diameter. The curve 2 correspond of the film prepared by sputtering the same target with gradient of $\mathrm{Gd}$ but by the use new methods (5). The curve 3 shows the compensation 


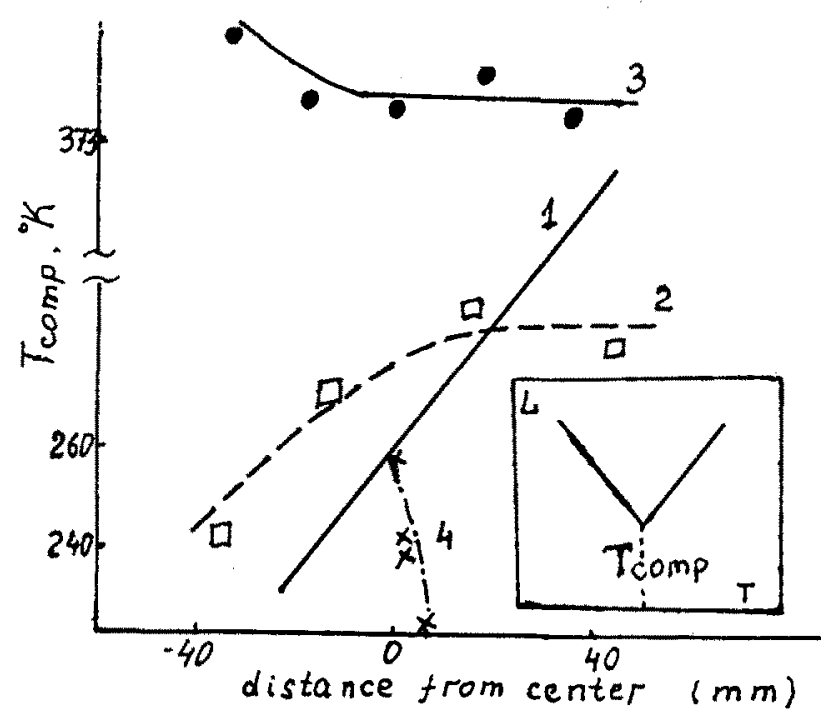

Fig. 1. The dependence of compensation temperature as a fumction the radial position. Insert-dependence of torque moment as a finction of the temperature.

temperature in the film GdCoTb. The target was with gradient of $\mathrm{Tb}$ and sputtered by use our methods. The dependence of $T_{\text {comp }}$ as position of the center substrate (curve 4) obtained in Ref. (9) is shown here too. $T_{\text {comp }}$ was determined by minimum $L(T)$ and is shown in the insert.

\section{RESULTS and DISCUSSION}

The temperature dependence of the torque moment is presented in fig. 2 for the Gd-Co multilayer films.

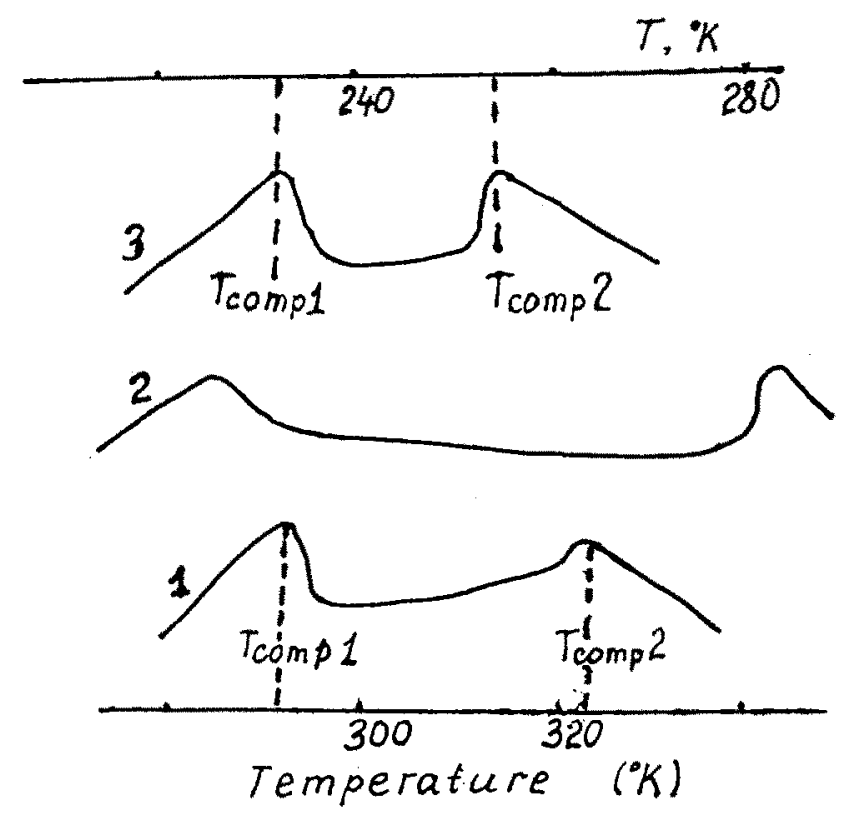

Fig. 2 The dependenco of torque moment a function of the temperature for the films prepared two different methods
It was assumed that for the double layer films with the different middle $T_{\text {coen }}$ of the layer it must be two minimums on the dependence $L(T)$. Really, as it is at fig 2 . there are just two minimums. The change of the position minimums at the curve L(T) obtained for different samples by the same magnetic field can be connected only with the change of the composition in the layers. The curve 2 and 3 correspond to the two-layer films obtained by new method (5) and show a possibility of the preparation the layers with different composition. The curves in fig. 2 are obtained at the magnetic field of $5 \mathrm{kOe}$. The curve 1corresponds to the film deposited by means of bias voltage applied to the substrates. As it is shown in Fig 3: this film consists of the five alliterating layers with composition by Co or Gd enriched.

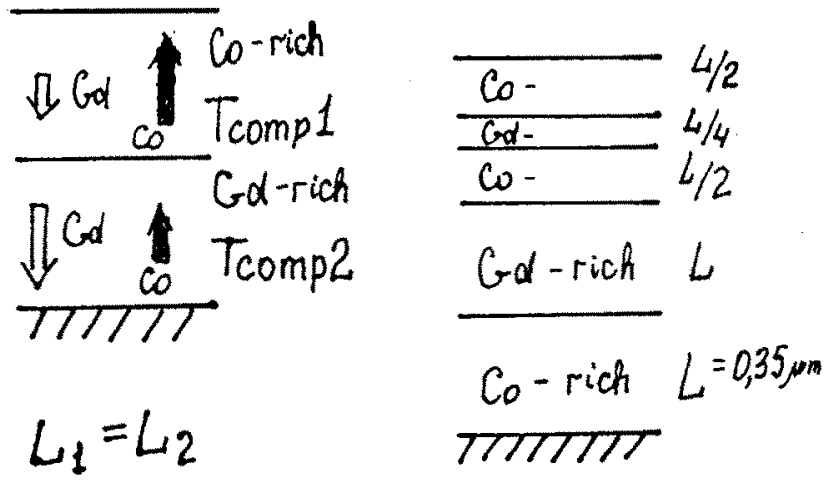

Fig 3 The two types of the structure of studied fitms

As is evident clear from the curve 1 the five-layer film behaves as two-layer films in the magnetic respect. At the same time in every layer of the multilayer structure in a magnetic field can be appear an other layer because of the inevitable presence of the composition gradient along thickness layers and a formation of the horizontal compensation domain wall. The behavior of the net magnetization in the layer is shown in fig 4. Consequently we can be aroused a magnetic structure of the multilayer film as sum of two double layers. In order to distinguish two sublayers connected with origin compensation wall from layers having another middle " $T_{\text {comp }}$ " every layer can be mark as $1 \mathrm{gr}$ or $2 \mathrm{gr}$ accordingly.

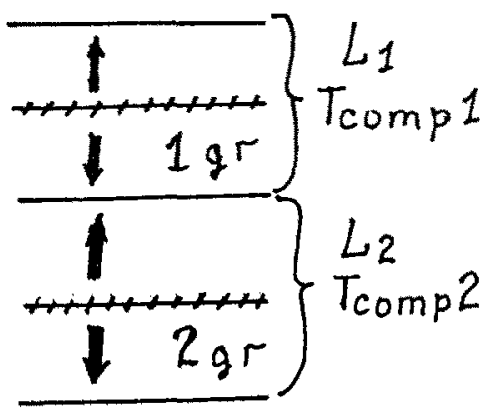

Fig 4 Schematic illustration of the behavior of net magnetization five layers films. 


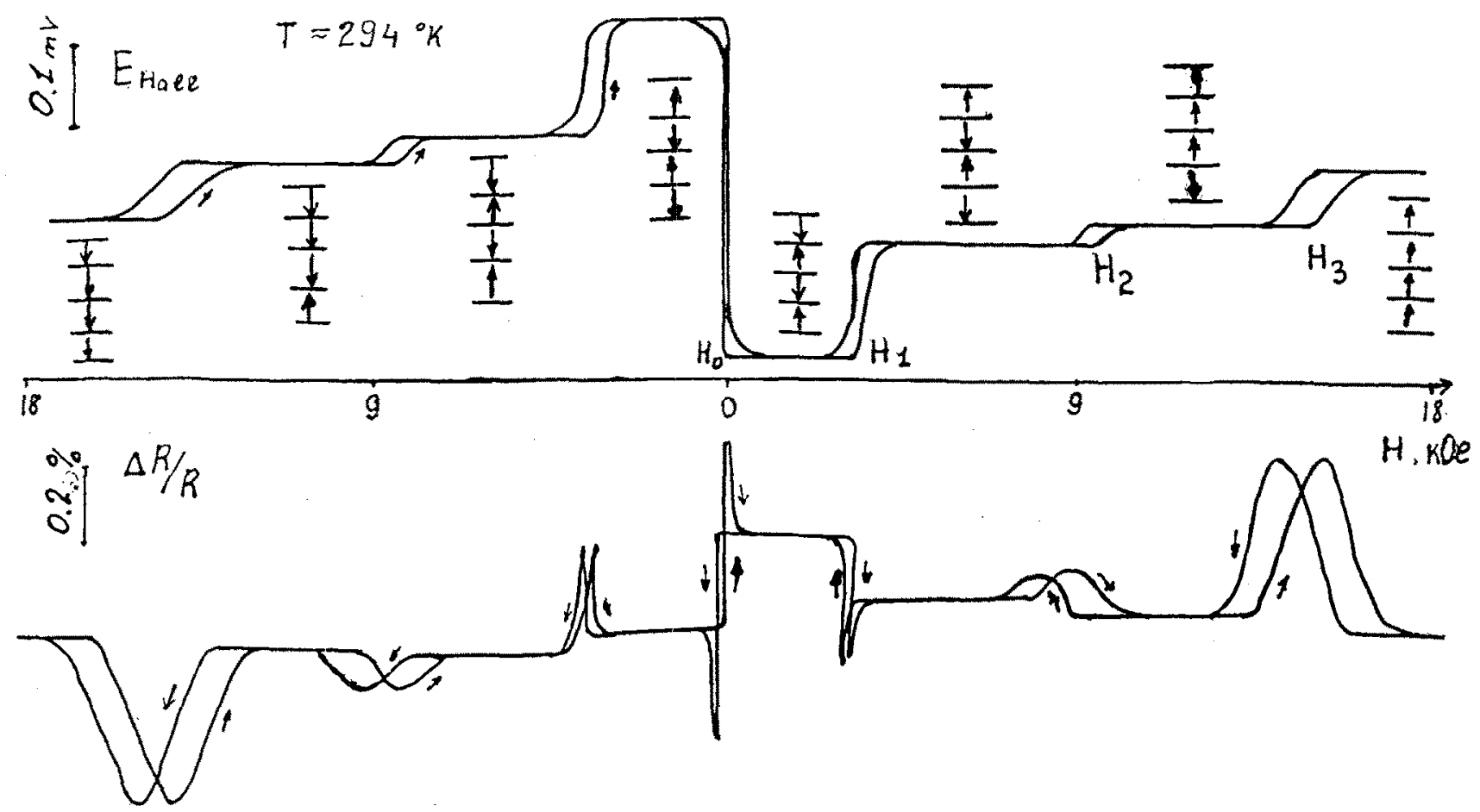

Fig. 5 The typical hysteresis loops of the Hall effoct and magnetaresistanco

The magnetization reversal process of these films was investigated in the temperature interval including the middle supposed "Toomp 1,2 ". Fig S. shows the typical Hall effect and MR hysteresis loops measured at the temperature between "T obvious clear from presented hysteresis loops we observed no simple behavior of the galvanomagnetics effects with the change of magnitude magnetic field. As it is shown in fig.5 there are three satellites hysteresis loops except the basic loop.

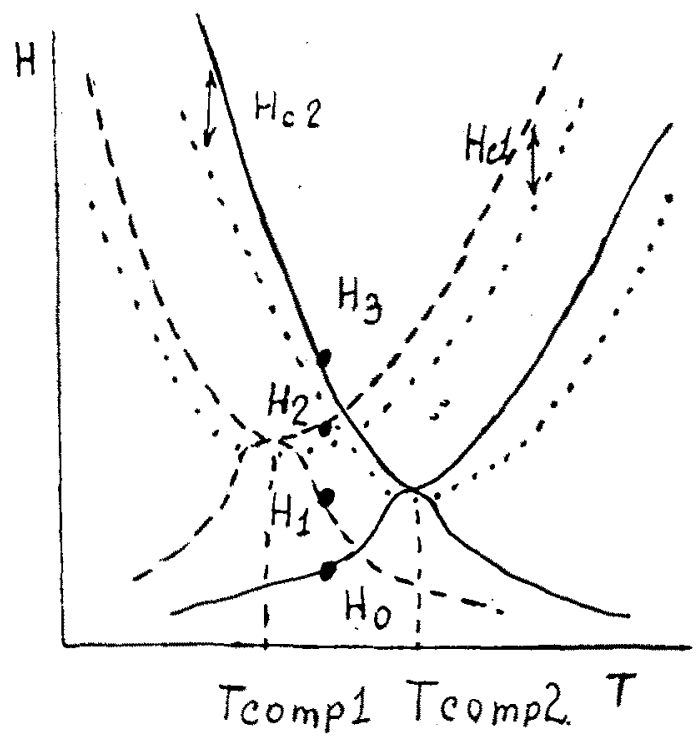

Fig 6 The H-T diagram of the five-layer film.
Their appearance can be understand on the band of the magnetic structure scheme (fig.4) and a H-T diagram of the magnetic state. The possible scheme of magnetic reversal process of the layers is presented in Fig.5. According to H-T diagram (11) if the film has the composition gradient, than another supplementary hysteresis loop and the corresponding characteristic switching field are observed. In our case we have a presence of second layer with his composition gradient and middle Tcomp 2. Consequently, two satellite loops arise. Therefore, on the base of our supposition and the literature dates $(11,12)$ the accordingly H-T diagram (fig 6) can be proposed. It is clear from diagram that there are four switching fields $\mathrm{H}_{0}, \mathrm{H}_{1}$ , $\mathrm{H}_{2}$ and $\mathrm{H}_{3}$ for this film at $\mathrm{T}_{\text {compl }}<\mathrm{T}<\mathrm{T}_{\text {comp } 2}$. These switching fields are shown also in the hysteresis loops of the Hall effect and magnetoresistance. The field $\mathrm{H}_{0}$ and $\mathrm{H}_{1}$ correspond probably to the basic magnetic reversal process of the layers with different compensation temperatures

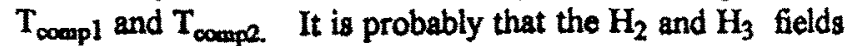
correspond to the addition magnetization reversal process of these layers. The magnetizations of the $1 \mathrm{gr}$ and $2 \mathrm{gr}$ layer line up along the direction of the magnetic field in sufficiently great magnitude of the fieid. It should be noted that if the orientation of magnetization changes in the layer than additional loops on the Hall hysteresis loop arise and electrical resistance's changes on the hysteresis loop of magnetoresistance. The MR hysteresis loops with the switching field $\mathrm{H}_{2}$ and $\mathrm{H}_{3}$ are like such satellite loops obtained earlier in Ref.13. It is confirmed by the magnetization reversal process in the $\operatorname{lgr}$ and 2 gr layer. The summary thicknesses of the layers are not equal to each 
other their amplitude of the satellite hysteresis loops are no equal.

We used double layers model with horizontal compensation domen wall but possibly the application of the model with oblique compensation wall (14). Also undoubtedly the simple H-T diagram (Fig.6) does not take consideration of exchange coupling between $\mathrm{Co}$ and $\mathrm{Gd}$ enriched layer therefore the real diagram will differ from presented one. The giving H-T scheme can be complete in the result more the detail investigation. More detailed reports on this will published.

\section{REFERENCES}

[1]. M.Kaneko, IEEE Trans. Magn, 28 (5), 2494 (1992)

[2].S.Esho, Jpn. J. Appl. Phys. 15. 93 (1976)

[3].Thu Chen, R.Malmhall and G.B.Charlan, J. Appl. Phys, 53,2356 (1982)

[4].T.Tokunada et all, IEEE Trans .Magn, vol Mag-22 ,N 5, September 1986
[5]. A.N.Sorokin and A.V.Svalov, Patent of Russia, 210202, C23 C14/32 (filed: 14.01.91, data of patent 29.09.93. to be publish

[6]. A.N Sorokin and A.V.Svalov, Claim for invention 4705561/21 (USSR) Date of positive resolution August 22.1990 , to be publish.

[7] V.O. Vas'kovskij et all, Phys.Met.Metal, 59(3),46 (1985)

[8] K.Okamoto and N.Miura, Physica B, 155, 259 (1989).

[9] Y.Togami and K.Kobayashi,Jpn.J.of Appl. Phys, 20,N8, 1457(1981).

[10] H.Tsujimoto and Y.Sakurai, Jpn.J.of Appl.Phys,22(12) 1845 (1983).

[11] V.I.Smirnov et all, Solid State Phys, 24 (1),314 (1982). [12]T.Kobayashi et all, Jpn.J of Appl.Phys, 20 (11), 2089 (1981).

[13] Pampura Ye.M, A.V.Svalov and A.N.Sorokin, Reports to 4 th All-union seminar on Magnetic Electronics, Krasnoyarsk, 106 (1990).

[14] M.Y.Bistrov and all, Solid State Physics 18 (1), 851 (1976). 